\title{
Editorial: Open Science and Ethics
}

\section{Marcus Düwell ${ }^{1}$}

Accepted: 16 December 2019/Published online: 31 December 2019

(C) Springer Nature B.V. 2019

Academic institutions in Europe (e.g. universities, research funding institutions, academies) are increasingly committing themselves to 'open science' principles (see e.g. http://openscienceasap. org/open-science/). Those principles are increasingly seen as the basis of good academic practice. They include a broad spectrum of considerations. On a primary level they include principles like 'transparent documentation of methodology' and 'peer review' that are already well-established elements of academic practices. On a secondary level they have to do with access to research (use of open sources and open access): access not only to the results of research, but also to underlying information, such as the empirical data on which research results are based. On a further level open science refers to a research culture in which research is communicated in ways that are not only accessible to specialists in a field. Open science principles require that such communication with various societal stakeholders should not only be a one-way communication (academics inform the public about their research results) but instead should be a two-way communication, in which relevant stakeholders should have significant impact on the formulation of research questions. Research processes should in this view be increasingly seen as responsive to public discussions and public interests. The role of research can be summarized as supporting society in understanding and solving societal challenges.

The debate on 'open science' is at the moment primarily located on the level of funding bodies and university boards. But increasingly institutions have declared that they will organise their funding schemes according to open science principles and in some countries (like the Netherlands) there are discussions about whether appointments and promotions of academic staff should take into consideration to what extent the staff has shown their commitment and ability to confirm to those principles. And the strong support in Europe for Open Access (https://www.coalition-s.org/) has to be seen in this context as well. The protagonists of Open Science see Open Access as part of a more general change of research strategies.

At first sight it seems to be self-evident that the ethics-community should embrace those open science principles unconditionally. Transparency, accessibility, and solving societal challenges are laudable goals. Understanding and solving societal challenges seems to be

Marcus Düwell

m.duwell@uu.nl 
the core business of ethics: the theoretical branches of ethics aim for a better understanding of the practical and moral dimension of human life in general and applied ethics engages with possible solutions of urgent societal challenges. Thus, 'open science' seems to be the ideal context for ethical research to flourish. But as philosophers we should be a bit more reflective than just preaching the gospel of open science without critical remarks. In the following I want to highlight five possible pitfalls and problems around open science. The aim is not to frustrate the entire enterprise but to contribute to a responsible way of introducing the open science principles.

First, some formulations of open science principles seem to presuppose that all research that conforms to open science principles should be directly responsive to societal challenges. In that sense open science seems to be opposed to 'blue skies research' or 'curiosity driven research'. I think those oppositions are misconceived. Research is always 'curiosity driven'. Its outcome is always open, otherwise it's not worth pursuing. At the same time, research is always contributing to the better understanding of relevant aspects of the natural or the human world. But the type of societal impact research can be very different. Research can be directly or indirectly relevant for our understanding of societal challenges. The impact can be more or less predictable. The impact can be short term or long term. It would be a misunderstanding of open science if one would assume that direct, predictable, and short-term impacts on our understanding and solution of societal challenges would be preferable to indirect, uncertain, or unpredictable and long-term impacts. That holds for research in general, but it also holds for ethical research. It is possible that an interpretation of the ethics of Seneca or a phenomenological analysis of perception is all-things-considered more important for the understanding of societal challenges than an article on business ethics. Thus, the claim that open science should be responsive to societal challenges should develop a nuanced understanding of the different forms of impact that different research activities can have for the understanding and solution of societal challenges.

Second, this has some implications for the type of research activities open sciences advocates should embrace. In that context it's often claimed that research must be open and directly accessible for a broader academic community or even to society at large. In this generality the claim is problematic and not ethically acceptable. If it were correct, it would make specialized research with technical terminology illegitimate. Nobody should embrace such a strategy. One can, however, expect that every researcher reflects on the place of his or her specialized research within the broader scheme of things. Researchers should be capable of seeing the role and importance of their specialized research in a broader academic and societal context. If they are not capable of such a reflection, something has gone wrong. The increase of specialisation makes this task more demanding. Open science researchers should be worried about the capacity of the academic community to self-reflectively relate specialized research to a broader academic horizon, and they should be concerned with how to create an infrastructure that makes such self-reflection possible. Thus, specialisation should not be opposed to open science principles. But open science should reflect on the necessary academic structures for enabling critical self-reflection that relates specialized research to broader questions of understanding of societal challenges.

Three, some open science advocates see it as necessary that research in line of open science principles engage directly with societal actors and stakeholders. And indeed, if a bioethicist would never speak to physicians, if a business-ethicists would never engage with managers, if a sociologist of technology would never engage with designers of technologies, this would be strange. But this only holds for parts of research community. Would this principle be generally 
valid, research in Celtic language could never be legitimate because the relevant stakeholders died some centuries ago. But if we wouldn't continue this research, we would lose our capacity to understand this culture that had an important impact on European history. We cannot know under which conditions this knowledge will be relevant in the future. Only if we would assume a linear, teleological theory of knowledge development can we exclude the possibility that this knowledge could become societally relevant. Thus, stakeholder engagement is a very important strategy for some research domains, but if it is claimed to be a universal requirement of research according to open science principles then it is certainly problematic.

Fourth, it sometimes sounds as if open science is opposed to academic freedom. The claims for autonomy of research and academic freedom seems to be notions of the 'old university', a university that defends its privileges of un-detached reflection against the demand to engage with societal challenges. I think the opposition between academic freedom and open science is a misconception. Academic freedom is a necessary requirement for any research. Researchers should be allowed to produce research results that nobody wants to hear. If that is not possible, research is worthless. In the 19th and early twentieth Century the great threat to academic freedom was the interference of state or church in the inner logic of the research process. Nowadays, interference by industry and particular societal groups form a much more serious threat to academic freedom. Thus, it is inherently problematic if open science principles were opposed to academic freedom.

Fifth, if we assume that research should be responsive to societal questions, it seems that the research question comes from society and that academia is responsible for providing the answer. I think that this is conceptually misconstrued. Formulating one's own research question is the most important thing a researcher can do. Formulating a research question already determines which disciplines are relevant for answering this question. We can even go further. It is a characteristic of the university as it was developed since the Middle Ages that this university perceived itself as disciplinarily organised based on different questions, which the different disciplines aim to answer. You can look to nature, to society, to law etc. from a biological, sociological, historical etc. perspective, from very different angles and depending on one's research question a totally different discipline is appropriate for providing an answer. No institution and no voice in society can relieve the researcher of formulating the research question him- or herself. This also implies that no researcher can hide him- or herself behind the research community. Thus, the researcher or a group of researchers is responsible for the formulation of the research question.

To conclude, all of this can be terribly relevant. It is quite likely that in the future research funding will depend on commitment to open science principles. Conforming to open science principles may be decisive for the success of grant applications. So far, the open science principles are quite underdetermined. It will be the task for the years to come to specify those principles. This short editorial has tried to provide some orientation how we could interpret the open science principles and which pitfalls should be avoided. To avoid a misunderstanding: I really think that open science can be an opportunity for current universities. But I think that the specification of this principles is an important task. I have tried to formulate some challenges, and I think that it will be the task of the years to come to resolve them. I think it would be an important task for philosophers to actively contribute to this discussions.

Acknowledgements Thanks to Frank Midema, Vice-rector research of Utrecht University, for the inspiration for this editorial. 